\title{
Genome Sequence of Avirulent Riemerella anatipestifer Strain RA-SG
}

\author{
Jianfeng Yuan, Linlin Li, Minhua Sun, Jiawen Dong, Qilin Hu \\ Institute of Veterinary Medicine, Guangdong Academy of Agricultural Sciences, Guangzhou, China, and Guangdong Open Laboratory of Veterinary Public Health, \\ Guangzhou, China
}

\begin{abstract}
Riemerella anatipestifer is a pathogenic bacterium that has spread all over the world and is associated with epizootic infections in waterfowl and other avian species. $R$. anatipestifer RA-SG is an avirulent strain, isolated from an infected duck in Guangdong province, China. The genome sequence of this species is presented herein.
\end{abstract}

Received 25 December 2012 Accepted 7 January 2013 Published 7 March 2013

Citation Yuan J, Li L, Sun M, Dong J, Hu Q. 2013. Genome sequence of avirulent Riemerella anatipestifer strain RA-SG. Genome Announc. 1(2):e00218-12. doi:10.1128/ genomeA.00218-12

Copyright $\odot 2013$ Yuan et al. This is an open-access article distributed under the terms of the Creative Commons Attribution 3.0 Unported license.

Address correspondence to Jianfeng Yuan, jfeng.yuan@gmail.com, or Qilin Hu, hql562713@163.com.

$R$

iemerella anatipestifer, a Gram-negative, gliding-motile, and non-spore-forming rod-shaped bacterium, is the etiological agent of a contagious septicemic disease in waterfowl and other avian species $(1,2)$; according to $16 \mathrm{~S}$ rRNA gene analysis, it belongs to the family Flavobacteriaceae $(3,4)$. Up to now, at least 21 serotypes have been identified by tube agglutination and agar gel precipitin tests, and no cross-immunoprotection has been reported $(5,6)$. Based on public papers, three genome sequences of $R$. anatipestifer have been reported until now, those of strains ATCC 11845, RA-GD, and RA-YM (7-10). All of them are highly virulent to the susceptible hosts. Here, we sequenced the genome of $R$. anatipestifer RA-SG, an avirulent strain. We believe that it will be very helpful in understanding further the pathogenesis of R. anatipestifer.

The genome sequence was determined using an Illumina genome analyzer (0.5-kb and 2-kb paired-end libraries), with sequencing carried out by the Beijing Genomics Institute (BGI) in Shenzhen, China. The sequence reads were assembled into a total of 75 contigs ( $\mathrm{N}_{50}$ length, 224,073 bp) distributed over 31 scaffolds ( $\mathrm{N}_{50}$ length, 436,370 bp) using Short Oligonucleotide Alignment Program (SOAP)denovo. Glimmer 3.0 was used to predict the protein-coding sequences (CDSs) (11). The tRNAs, rRNAs, and small RNAs (sRNAs) were identified using tRNAscan, rRNAmmer, and Rfam, respectively (12-14). Genome annotation was generated by searching against the NCBI nonredundant (NR), Swiss-Prot, TrEMBL, Clusters of Orthologous Groups (COGs), and Kyoto Encyclopedia of Genes and Genomes (KEGG) databases $(15,16)$.

The genome sequence of avirulent $R$. anatipestifer RA-SG comprises $2,172,634 \mathrm{bp}$, with an average $\mathrm{G}+\mathrm{C}$ content of $34.94 \%$ and a coding percentage of 90.37 . The genome contains 2,072 putative open reading frames with an average length of $948 \mathrm{bp}$; it also contains 36 tRNA genes, 4 rRNA genes, and 9 sRNAs, constituting $90.37 \%$ of the genome. Of the CDSs, $37.55 \%$ could be assigned to COG families, and 959 genes were designated to 129 KEGG pathway mappings using KEGG Automatic Annotation Server (KAAS) (17). The repeat sequence length of 16,601 bp was determined by the RepeatMasker, RepeatProteinMasker, and Tandem Repeats Finder software programs. In addition, there are two credible clustered regularly interspaced short palindromic repeats (CRISPRs) and two possible CRISPRs, but there is no prophage sequence in the genome. Except for the general secretory $(\mathrm{Sec})$ and twin-arginine translocation (Tat) pathways, the classic secretion systems in the Gram-negative bacteria were not identified, which has been reported in other virulent $R$. anatipestifer strains. Moreover, three genomic islands were identified using SIGI-HMM; these were 4,772 bp, 3,537 bp, and 8,289 bp.

Overall, the genome sequence of avirulent $R$. anatipestifer RA-SG will provide the basis for a better understanding of the molecular pathogenesis of $R$. anatipestifer using comparative genomics.

Nucleotide sequence accession numbers. This Whole Genome Shotgun project has been deposited at DDBJ/EMBL/ GenBank under the accession no. ANGF00000000. The version described in this paper is the first version, ANGF01000000.

\section{ACKNOWLEDGMENTS}

This work is supported by the Young Scientists Fund of the National Natural Science Foundation of China (grant no. 3110183 to Jianfeng Yuan), Guangdong Natural Science Foundation (grant no. 9251064001000010 and 10151064001000013 to Jianfeng Yuan), Guangdong Science and Technology Project (no. 2012A020100001), and President Foundation of Guangdong Academy of Agricultural Sciences (grant no. 201015 to Jianfeng Yuan).

\section{REFERENCES}

1. Sandhu TS. 2008. Riemerella anatipestifer infection, p 758-764. In Saif YM (ed), Diseases of poultry, 12th ed. Blackwell Publishing, Oxford, United Kingdom.

2. McBride MJ, Zhu Y. 2013. Gliding motility and Por secretion system genes are widespread among members of the phylum Bacteroidetes. J. Bacteriol. 195:270-278.

3. Segers P, Mannheim W, Vancanneyt M, De Brandt K, Hinz KH, Kersters K, Vandamme P. 1993. Riemerella anatipestifer gen. nov., comb. nov., the causative agent of septicemia anserum exsudativa, and its phylogenetic affiliation within the Flavobacterium-Cytophaga rRNA homology group. Int. J. Syst. Bacteriol. 43:768-776.

4. Subramaniam S, Chua KL, Tan HM, Loh H, Kuhnert P, Frey J. 1997. Phylogenetic position of Riemerella anatipestifer based on 16S rRNA gene sequences. Int. J. Syst. Bacteriol. 47:562-565.

5. Pathanasophon P, Tanticharoenyos T, Sawada T. 1994. Physiological 
characteristics, antimicrobial susceptibility and serotypes of Pasteurella anatipestifer isolated from ducks in Thailand. Vet. Microbiol. 39:179-185.

6. Pathanasophon P, Sawada T, Tanticharoenyos T. 1995. New serotypes of Riemerella anatipestifer isolated from ducks in Thailand. Avian Pathol. 24:195-199.

7. Mavromatis K, Lu M, Misra M, Lapidus A, Nolan M, Lucas S, Hammon N, Deshpande S, Cheng JF, Tapia R, Han C, Goodwin L, Pitluck S, Liolios K, Pagani I, Ivanova N, Mikhailova N, Pati A, Chen A, Palaniappan K, Land M, Hauser L, Jeffries CD, Detter JC, Brambilla EM, Rohde M, Göker M, Gronow S, Woyke T, Bristow J, Eisen JA, Markowitz V, Hugenholtz P, Klenk HP, Kyrpides NC. 2011. Complete genome sequence of Riemerella anatipestifer type strain (ATCC 11845). Stand. Genomics Sci. 4:144-153.

8. Wang X, Zhu D, Wang M, Cheng A, Jia R, Zhou Y, Chen Z, Luo Q, Liu F, Wang Y, Chen XY. 2012. Complete genome sequence of Riemerella anatipestifer reference strain. J. Bacteriol. 194:3270-3271.

9. Yuan J, Liu W, Sun M, Song S, Cai J, Hu S. 2011. Complete genome sequence of the pathogenic bacterium Riemerella anatipestifer strain RAGD. J. Bacteriol. 193:2896-2897.

10. Zhou Z, Peng X, Xiao Y, Wang X, Guo Z, Zhu L, Liu M, Jin H, Bi D, Li Z, Sun M. 2011. Genome sequence of poultry pathogen Riemerella anatipestifer strain RA-YM. J. Bacteriol. 193:1284-1285.
11. Delcher AL, Bratke KA, Powers EC, Salzberg SL. 2007. Identifying bacterial genes and endosymbiont DNA with Glimmer. Bioinformatics 23:673-679.

12. Lowe TM, Eddy SR. 1997. tRNAscan-SE: a program for improved detection of transfer RNA genes in genomic sequence. Nucleic Acids Res. 25: 955-964.

13. Lagesen K, Hallin P, Rødland EA, Staerfeldt HH, Rognes T, Ussery DW. 2007. RNAmmer: consistent and rapid annotation of ribosomal RNA genes. Nucleic Acids Res. 35:3100-3108.

14. Griffiths-Jones S, Bateman A, Marshall M, Khanna A, Eddy SR. 2003. Rfam: an RNA family database. Nucleic Acids Res. 31:439-441.

15. Tatusov RL, Fedorova ND, Jackson JD, Jacobs AR, Kiryutin B, Koonin EV, Krylov DM, Mazumder R, Mekhedov SL, Nikolskaya AN, Rao BS, Smirnov S, Sverdlov AV, Vasudevan S, Wolf YI, Yin JJ, Natale DA. 2003. The COG database: an updated version includes eukaryotes. BMC Bioinformatics 4:41.

16. Kanehisa M, Goto S. 2000. KEGG: Kyoto encyclopedia of genes and genomes. Nucleic Acids Res. 28:27-30.

17. Moriya Y, Itoh M, Okuda S, Yoshizawa AC, Kanehisa M. 2007. KAAS: an automatic genome annotation and pathway reconstruction server. $\mathrm{Nu}-$ cleic Acids Res. 35:W182-W185. 